\title{
An Insight into Coherent Backscattering Evaluation Techniques of Prospective Use in High Frequency Polarimetric Radar Image Processing
}

\begin{abstract}
J. KAPELEWSKI*
Faculty of Electronics, Military University of Technology, S. Kaliskiego 2, 00-908 Warsaw, Poland

Coherence in multiple scattering is important as it applies to imaging and detection of object in multiple scattering environments. These phenomena may also be used to investigate images of objects obscured by them. The scattering observations using very rough surfaces can provide useful insight into their statistic as they lie outside the range of validity of the standard theories. Recently, there has been growing interest in the phenomenon of backscattering enhancement in the reflection of electromagnetic waves of $\mathrm{mm}$ wave range from both atmospheric hydrometeors (rain, snow and precipitation) as well as very rough surfaces (including that of sea and terrain sea). Strong backscattering enhancement of $\mathrm{mm}$ waves incident at different incident angles, both in the TE and TM polarizations, was observed experimentally. In the paper a short description of specific techniques used for treating image processing of strongly irregular media is shortly presented. An emphasis is placed on the phenomena of interest in advanced polarimetric radar especially in $\mathrm{mm}$ wave range.
\end{abstract}

PACS numbers: 41.20.Jb, 03.50.De

\section{Backscattering enhancement}

Backscattering enhancement phenomena have been observed for many years. It has been sometimes called the "retroreflectance" or the "opposition effect".

Recently, more quantitative experimental and theoretical studies of the enhancement have been reported. Watson in [1] noted that the backscattered intensity is twice the intensity of the sum of incoherent multiple scattering and the first-order scattering. De Wolf in [2] showed that the backscattered intensity from turbulence is proportional to the fourth-order moment and approximately twice the multiple scattered intensity.

It was explained in [3] that the enhanced peak is caused by the constructive interference of two waves traversing through the same particles in opposite directions. Physicists have also recognized that the transport of electrons in a strongly disordered material is governed by multiple scattering and that multiple scattering leads to "weak Anderson localization" caused by "coherent backscattering". It is then shown that both electron localization in disordered material and electromagnetic (EM) wave localization in disordered materials are governed by coherent backscattering $(\mathrm{CB})$ which is caused by the constructive interference of two waves traversing in opposite directions. One $I_{l}$ corresponds to the wave multiply scattered through many particles, called the "ladder term". The other $I_{\mathrm{c}}$ corresponds to two waves traversing through the

* corresponding author; e-mail:

jerzy.kapelewski@wel.wat.edu.pl same particles in opposite directions. This is called the "cyclical" or the "maximally crossed" term and has the same magnitude as $I_{l}$ in the back direction, but diminishes away from the back direction (Fig. 1). The essence of this division will be highlighted in Sect. 3 .

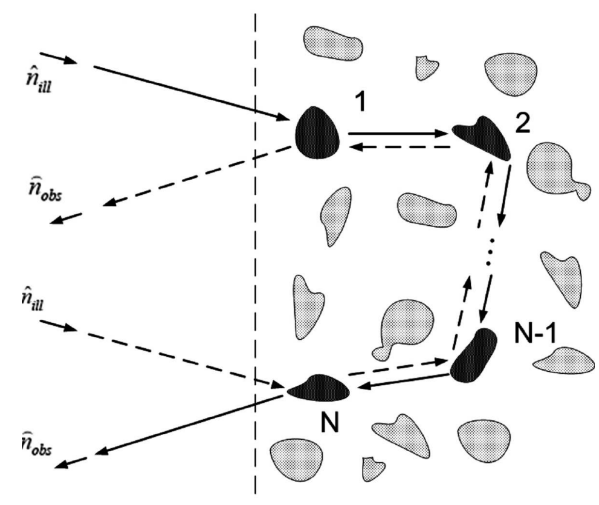

Fig. 1. Schematic explanation of CB. The direct (solid arrows) and reverse (dashed arrows) wave paths go through the same group of $N$ particles, but in opposite directions.

Also two waves scattered off the sloped surface interfere constructively in the back direction producing the enhanced peak. The angular width is relatively broad and approximately proportional to the slope (Fig. 2).

A simple picture of the scattering process leads to the conclusion that the backscatter peak is caused by multiple scatter paths. From Fig. 2 the scattering paths $A B C$ and $C B A$ give scattered waves with a difference in phase $\left(\boldsymbol{k}_{1}+\boldsymbol{k}_{2}\right) \cdot \boldsymbol{d}$. This term is zero if $\boldsymbol{k}_{2}=-\boldsymbol{k}_{1}$ which is 


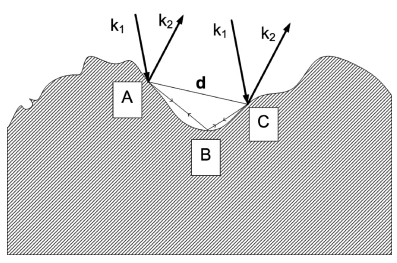

Fig. 2. The geometry of the scattering process time reversed partner $C B A$ of the direct path $A B C$ [4].

exactly the case for backscatter (see Sect. 3). Thus the backscatter terms add coherently to give a factor of two larger intensity in this direction than in other directions which have incoherently added terms. The width of the backscatter peak can also be estimated from this simple model. The coherent term will give no contribution when the scattered waves are essentially out of phase. The half width of the peak is then found to be [4]:

$$
\theta_{1 / 2} \approx \frac{\lambda}{\langle d\rangle}, \quad d=|\boldsymbol{A C}| .
$$

Another enhancement occurs when the root mean square (rms) of the surface height is much smaller than a wavelength, but the second medium supports a surface wave. This occurs when an optical beam is scattered from a slightly rough metallic surface. If the incident wave is $p$-polarized (parallel to the plane of incidence) and the dielectric constant of the second medium has a negative real part, then a surface wave is excited on the surface and two surface waves traversing on the surface in opposite directions interfere constructively in the back direction, producing the enhancement [5].

\section{Wave scattering from random media}

Following [5] let us consider electromagnetic (EM) beam propagating through turbulent air. The reflective index $n(\boldsymbol{r}, t)$ of air varies randomly in space and time, and therefore, the amplitude and the phase of the wave also vary randomly in space and time.

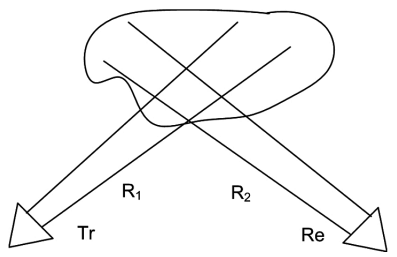

Fig. 3. Scattering by random cloud of scatterers.

Suppose that a time-harmonic field with $\exp (-\mathrm{i} \omega t)$ is incident on the medium. If we take a component $E_{x}$, of the field vector $\boldsymbol{E}$, the scalar field $u(\boldsymbol{r}, t)=E_{x}$, is a random function of position $r$ and time $t$. We write $u$ as follows:

$$
u(\boldsymbol{r}, t)=\operatorname{Re}(U(\boldsymbol{r}, t)) \exp (-\mathrm{i} \omega t),
$$

where $U(\boldsymbol{r}, t)=A(\boldsymbol{r}, t) \exp (-\mathrm{i} \phi(\boldsymbol{r}, t))$ is called the com- plex envelope and $A$ and $\phi$ are the random functions.

Now we write $U$ as the sum of the coherent field $\langle U\rangle$ and the fluctuating field $U_{\mathrm{f}}$ :

$$
U(\boldsymbol{r}, t)=\langle U(\boldsymbol{r}, t)\rangle+U_{\mathrm{f}}(\boldsymbol{r}, t)
$$

where \langle\rangle denotes the ensemble average.

In theoretical work, we normally consider the ensemble average, but in practice, this can often be approximated by its spatial or time average (ergodic principle).

The field $U$, neglecting the cross polarization, satisfies the scalar wave equation

$$
\left[\nabla^{2}+k^{2}\left(1+\varepsilon_{1}\right)\right] U=0
$$

where the dielectric constant $\varepsilon(\boldsymbol{r}, t)=n^{2}(\boldsymbol{r}, t)$ is a random function and

$$
\varepsilon=\langle\varepsilon\rangle\left(1+\varepsilon_{1}\right), \quad k^{2}=\frac{\omega^{2}}{c^{2}}\langle\varepsilon\rangle .
$$

$\varepsilon_{1}$ is the fluctuation and is assumed to be small.

Let us first consider the coherent field $\langle U\rangle$. From (2.3), we get

$$
\nabla^{2}\langle U\rangle+k^{2}\langle U\rangle+k^{2}\left\langle\varepsilon_{1} U\right\rangle=0 .
$$

Let us note that $\varepsilon_{1}$ and $U$ are correlated. The effective propagation constant $K$ for the coherent field is defined by

$$
\left(\nabla^{2}+K^{2}\right)\langle U\rangle=0 .
$$

An approximate expression for $K$ has been shown by $[6]$ :

$$
K^{2}=k^{2} \varepsilon_{\mathrm{e}}=k^{2}+k^{3} \int_{0}^{\infty} \exp (\mathrm{i} k r) \sin k r\left\langle\varepsilon_{1} \varepsilon_{2}\right\rangle \mathrm{d} r,
$$

where $\left\langle\varepsilon_{1} \varepsilon_{2}\right\rangle=\left\langle\varepsilon_{1}\left(\boldsymbol{r}_{1}\right) \varepsilon_{1}\left(\boldsymbol{r}_{2}\right)\right\rangle$ is the correlation function of $\varepsilon_{1}$ and is a function of the separation distance $r=\left|\boldsymbol{r}_{1}-\boldsymbol{r}_{2}\right|$.

The imaginary part of $K$ which represents the attenuation is given by

$$
K_{\mathrm{i}}=\frac{\pi^{2} k^{2}}{2} \int_{0}^{2 k} \Phi_{\varepsilon}\left(k_{s}\right) k_{s} \mathrm{~d} k_{s}
$$

where $\Phi_{\varepsilon}$ is the spectral density of the fluctuation $\varepsilon_{1}$ :

$$
\Phi_{\varepsilon}\left(k_{s}\right)=\frac{1}{(2 \pi)^{3}} \int\left\langle\varepsilon_{1} \varepsilon_{2}\right\rangle \exp \left(-\mathrm{i} \boldsymbol{K}_{s} \cdot \boldsymbol{r}\right) \mathrm{d} V .
$$

In $(2.8), 2 K_{\mathrm{i}}$ is equal to the scattering cross-section per unit volume of the random medium, representing the power scattered by the randomness. Note that $\varepsilon_{\mathrm{e}}$ in $(2.7)$ is the effective dielectric constant of the random medium and is, in general, complex even in a lossless medium. The imaginary part represents the attenuation due to scattering.

Let us next consider scattering by turbulent air (Fig. 3). We start with the radar equation for the case shown in Fig. 3. The received power $P_{\mathrm{r}}$ is given by

$$
P_{\mathrm{r}}=P_{\mathrm{t}} \frac{\lambda^{2}}{(4 \pi)^{3}} \int_{V_{\mathrm{c}}} \frac{G_{\mathrm{t}} G_{\mathrm{r}}}{R_{1}^{2} R_{2}^{2}} \sigma_{\mathrm{bi}} \exp \left(-\tau_{1}-\tau_{2}\right) \mathrm{d} V,
$$

where $\sigma_{\mathrm{bi}}$ is the bistatic scattering cross-section per unit volume of the turbulence and is given by the spectral density $\Phi_{\varepsilon}$ of the turbulence, and $\tau$ is the optical distance 


$$
\begin{aligned}
& \sigma_{\mathrm{bi}}=2 k^{4} \Phi_{\varepsilon}\left(k_{s}\right), \quad k_{s}=2 k \sin \left(\frac{\theta}{2}\right), \\
& \tau_{1}=\int_{0}^{R_{1}} 2 K_{\mathrm{i}} \mathrm{d} R,
\end{aligned}
$$

$K_{\mathrm{i}}$ is given in (2.8).

Let us note that the transmitted and the scattered waves attenuate through the turbulence with the attenuation constant of the coherent wave, and this process is called the first-order scattering or the distorted Born approximation. The cross-section $\sigma_{\mathrm{bi}}$ is proportional to the spectral density at the turbulent eddy size $l$,

$$
k_{s}=\frac{2 \pi}{l}=2 k \sin \left(\frac{\theta}{2}\right)
$$

representing the Bragg scattering. This simple expression (2.10) must be extended to include multiple scattering to explain backscattering enhancement. Even though most of the studies are directed to wave propagation in turbulence, similar techniques should be applicable to ultrasound scattering by tissues.

\section{Diagrammatic analysis}

Now, we present the way of backscattering evaluation using an approximation of Bethe-Salpeter equation. We will base on excellent formulation as given in [7,8]. One can write down the intensity Green function inside the disordered medium by means of Feyman-type diagram as

$$
\mathrm{gg}^{*}=\left[+{ }^{-x-}+\begin{array}{l}
-x-x-x-x \\
-x-x-x-x-x-x-x-\ldots
\end{array}+x\right.
$$

By convention the upper line denotes the amplitude and the lower line the complex conjugate amplitude. Dashed lines indicate the correlated scatterers. Since we average over the disorder, the number of scatterers runs from zero to infinity in between two scatterers that are connected with dashed lines from upper to lower line, for instance in the third diagram of Eq. (3.1). We can account for this at once by the use of the dressed Green function in between those two scatterers.

In this way they obtain at the Bethe-Salpeter equation in the diagrammatic form

$$
\begin{aligned}
& \left\langle g g^{*}\right\rangle=\square+\square\left\langle g g^{*}\right\rangle
\end{aligned}
$$

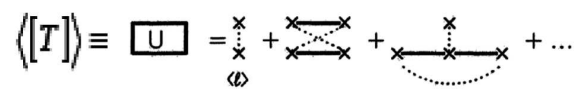

where $U$ is the so-called irreducible vertex. It is the intensity equivalent of the self energy from the Dyson equation. Iterating the Bethe-Salpeter equation to yields,

$$
\left\langle g g^{*}\right\rangle=G G^{*}+G G^{*} K G G^{*}
$$

which defines the reducible vertex $K$ as

$$
K=U+U G G^{*} K \text {. }
$$

In the weak-scattering regime all recurrent scattering events can be neglected, and we only take into account the first contribution to the irreducible vertex $U$,

$$
U=\langle L\rangle \equiv\left\langle\left[T_{\mathrm{L}}\right]\right\rangle
$$

where $\langle L\rangle$ is defined as the first diagram of Eq. (3.3). This leads to diffuse transport of light through the medium. However, in order to describe coherent backscattering of light, we also need the so-called most crossed (or cyclical) diagrams, describing the interference between time reversed waves.

Restricting ourselves only to these components one can approximate at the reducible vertex of two term sum

$$
K=\langle L\rangle+\langle C\rangle \equiv\left\langle\left[T_{\mathrm{L}}\right]\right\rangle+\left\langle\left[T_{\mathrm{C}}\right]\right\rangle
$$

with $\langle L\rangle$ the sum of all ladder diagrams and $\langle C\rangle$ the sum of the cyclical diagrams

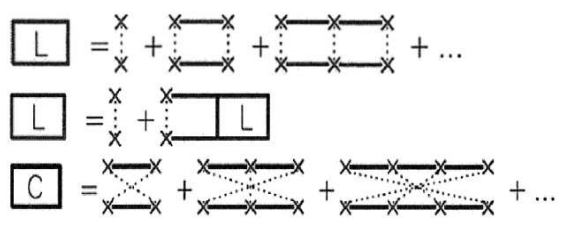

Equation (3.9) reads in real spaces coordinates

$$
\begin{aligned}
& \left\langle L\left(\boldsymbol{r}_{1}, \boldsymbol{r}_{2}, \boldsymbol{r}_{3}, \boldsymbol{r}_{4}\right)\right\rangle \\
& =n|t|^{2} \delta\left(\boldsymbol{r}_{1}-\boldsymbol{r}_{2}\right) \delta\left(\boldsymbol{r}_{1}-\boldsymbol{r}_{3}\right) \delta\left(\boldsymbol{r}_{1}-\boldsymbol{r}_{4}\right) \\
& \quad+n|t|^{2} \int \mathrm{d} \boldsymbol{r}_{5} \mathrm{~d} \boldsymbol{r}_{6} G\left(\boldsymbol{r}_{1}, \boldsymbol{r}_{5}\right) G^{*}\left(\boldsymbol{r}_{1}, \boldsymbol{r}_{5}\right) \\
& \quad \times\left\langle L\left(\boldsymbol{r}_{5}, \boldsymbol{r}_{6}, \boldsymbol{r}_{3}, \boldsymbol{r}_{4}\right)\right\rangle .
\end{aligned}
$$

Noting that the cyclical diagrams are quite similar to the ladder diagrams, except that the complex conjugate amplitude traverses the scattering sequence in reversed order, we expect $\langle L\rangle$ and $\langle C\rangle$ to fulfil a similar equation, if we separate out the single scattering contribution to $\langle L\rangle$. Hence, one can write for $\langle L\rangle$ :

$$
\begin{aligned}
& \left\langle L\left(\boldsymbol{r}_{1}, \boldsymbol{r}_{2}, \boldsymbol{r}_{3}, \boldsymbol{r}_{4}\right)\right\rangle \\
& \quad=n|t|^{2} \delta\left(\boldsymbol{r}_{1}-\boldsymbol{r}_{2}\right) \delta\left(\boldsymbol{r}_{1}-\boldsymbol{r}_{3}\right) \delta\left(\boldsymbol{r}_{1}-\boldsymbol{r}_{4}\right) \\
& \quad+F\left(\boldsymbol{r}_{1}, \boldsymbol{r}_{3}\right) \delta\left(\boldsymbol{r}_{1}-\boldsymbol{r}_{2}\right) \delta\left(\boldsymbol{r}_{3}-\boldsymbol{r}_{4}\right) .
\end{aligned}
$$

Inserting this equation in Eq. (3.11) one obtains an integral formula for $F\left(\boldsymbol{r}_{1}, \boldsymbol{r}_{3}\right)$ :

$$
\begin{aligned}
& F\left(\boldsymbol{r}_{1}, \boldsymbol{r}_{3}\right)=n^{2}|t|^{4}\left|G\left(\boldsymbol{r}_{1}, \boldsymbol{r}_{3}\right)\right|^{2} \\
& \quad+n|t|^{2} \int \mathrm{d} \boldsymbol{r}^{\prime}\left|G\left(\boldsymbol{r}_{1}, \boldsymbol{r}^{\prime}\right)\right|^{2} G^{*}\left(\boldsymbol{r}^{\prime}, \boldsymbol{r}_{3}\right) .
\end{aligned}
$$

The cyclical diagrams can then be summed to give

$$
\left\langle C\left(\boldsymbol{r}_{1}, \boldsymbol{r}_{2}, \boldsymbol{r}_{3}, \boldsymbol{r}_{4}\right)\right\rangle=F\left(\boldsymbol{r}_{1}, \boldsymbol{r}_{3}\right) \delta\left(\boldsymbol{r}_{1}-\boldsymbol{r}_{4}\right) \delta\left(\boldsymbol{r}_{2}-\boldsymbol{r}_{3}\right) .
$$

We only have to solve one integral equation to obtain both the contributions of the ladder diagrams and the cyclical diagrams. This is an essential reduction of the scattering problem.

The reflected and transmitted intensities are conveniently described by bistatic coefficients. A bistatic coefficient is the observed intensity in a certain direction for normalized incident intensity, and corrected for the distance to the scattering medium and the observed area $A$,

$$
\gamma\left(\mu_{i}, \mu_{s}\right) \equiv \frac{2 \pi r^{2}}{A} I(\boldsymbol{r})
$$

with $\mu_{i}=\cos \theta_{i}, \mu_{s}=\cos \theta_{s}$, and $\theta_{i}$ the direction of the 
incident light and $\theta_{s}$ - the direction of observation (both with respect to the surface normal pointing outwards).

According to [7] the intensity outside the scattering medium $I_{\text {out }}$ is given by

$$
\begin{aligned}
& I_{\mathrm{out}}(\boldsymbol{r})=\left|\Psi_{\mathrm{coh}}\left(\boldsymbol{r}_{1}\right)\right|^{2}+\int \mathrm{d} \boldsymbol{r}^{\prime} G\left(\boldsymbol{r}^{\prime}, \boldsymbol{r}\right) G^{*}\left(\boldsymbol{r}^{\prime}, \boldsymbol{r}\right) I\left(\boldsymbol{r}^{\prime}\right) \\
& \quad=\left|\Psi_{\mathrm{coh}}\left(\boldsymbol{r}_{1}\right)\right|^{2}+\int \mathrm{d} \boldsymbol{r}_{1} \mathrm{~d} \boldsymbol{r}_{2} \mathrm{~d} \boldsymbol{r}_{3} \mathrm{~d} \boldsymbol{r}_{4} G\left(\boldsymbol{r}, \boldsymbol{r}_{1}\right) G^{*}\left(\boldsymbol{r}, \boldsymbol{r}_{2}\right) \\
& \quad \times K\left(\boldsymbol{r}_{1}, \boldsymbol{r}_{2}, \boldsymbol{r}_{3}, \boldsymbol{r}_{4}\right) \Psi_{\mathrm{coh}}\left(\boldsymbol{r}_{3}\right) \Psi_{\mathrm{coh}}^{*}\left(\boldsymbol{r}_{4}\right) .
\end{aligned}
$$

The two Green functions transport the intensity inside the scattering medium towards the observation point $\boldsymbol{r}$.

From this, one can find for the reflection bistatic coefficient

$$
\begin{aligned}
& \gamma\left(\mu_{i}, \mu_{s}\right)=\frac{1}{8 \pi A} \int_{\text {slab }} \mathrm{d} \boldsymbol{r}_{1} \mathrm{~d} \boldsymbol{r}_{2} \mathrm{~d} \boldsymbol{r}_{3} \mathrm{~d} \boldsymbol{r}_{4} \\
& \quad \times \exp \left(-\left(\tau_{1}+\tau_{2}\right) / 2 \mu_{s}\right) \exp \left(\mathrm{i} \boldsymbol{k}_{s} \cdot\left(\boldsymbol{r}_{1}-\boldsymbol{r}_{2}\right)\right) \\
& \quad \times K\left(\boldsymbol{r}_{1}, \boldsymbol{r}_{2}, \boldsymbol{r}_{3}, \boldsymbol{r}_{4}\right) \exp \left(-\left(\tau_{3}+\tau_{4}\right) / 2 \mu_{i}\right) \\
& \quad \times \exp \left(-\mathrm{i} \boldsymbol{k}_{i} \cdot\left(\boldsymbol{r}_{3}-\boldsymbol{r}_{4}\right)\right)
\end{aligned}
$$

and for the transmission bistatic coefficient

$$
\begin{aligned}
& \gamma\left(\mu_{i}, \mu_{s}\right)=\frac{\exp \left(-b / \mu_{s}\right)}{8 \pi A} \int_{\text {slab }} \mathrm{d} \boldsymbol{r}_{1} \mathrm{~d} \boldsymbol{r}_{2} \mathrm{~d} \boldsymbol{r}_{3} \mathrm{~d} \boldsymbol{r}_{4} \\
& \quad \times \exp \left(\left(\tau_{1}+\tau_{2}\right) / 2 \mu_{s}\right) \exp \left(\mathrm{i} \boldsymbol{k}_{s} \cdot\left(\boldsymbol{r}_{1}-\boldsymbol{r}_{2}\right)\right) \\
& \quad \times K\left(\boldsymbol{r}_{1}, \boldsymbol{r}_{2}, \boldsymbol{r}_{3}, \boldsymbol{r}_{4}\right) \exp \left(-\left(\tau_{3}+\tau_{4}\right) / 2 \mu_{i}\right) \\
& \quad \times \exp \left(-\mathrm{i} \boldsymbol{k}_{i} \cdot\left(\boldsymbol{r}_{3}-\boldsymbol{r}_{4}\right)\right) .
\end{aligned}
$$

It is instructive to calculate separately the contributions to the reflected intensity of single scattering, the ladder and cyclical diagrams. For the single scattering bistatic coefficient $\gamma_{s}\left(\mu_{i}, \mu_{s}\right)$ the authors of [7] find

$$
\begin{aligned}
& \gamma_{s}\left(\mu_{i}, \mu_{s}\right) \\
& =\frac{1}{8 \pi A} \int_{\text {slab }} \mathrm{d} \boldsymbol{r}_{1} \mathrm{~d} \boldsymbol{r}_{2} \mathrm{~d} \boldsymbol{r}_{3} \mathrm{~d} \boldsymbol{r}_{4} n|t|^{2} \delta\left(\boldsymbol{r}_{12}\right) \delta\left(\boldsymbol{r}_{13}\right) \delta\left(\boldsymbol{r}_{14}\right) \\
& \quad \times \exp \left(-\frac{\tau_{1}+\tau_{2}}{2 \mu_{i}}-\frac{\tau_{3}+\tau_{4}}{2 \mu_{s}}\right) \\
& \times \exp \left(\mathrm{i} \boldsymbol{k}_{s} \cdot\left(\boldsymbol{r}_{1}-\boldsymbol{r}_{2}\right)-\mathrm{i} \boldsymbol{k}_{i} \cdot\left(\boldsymbol{r}_{3}-\boldsymbol{r}_{4}\right)\right) \\
& =\frac{A n|t|^{2} l_{\text {ex }}}{8 \pi A} \int_{0}^{b} \mathrm{~d} \tau_{1} \exp \left(-\tau_{1}\left(\frac{1}{\mu_{i}}+\frac{1}{\mu_{s}}\right)\right) \\
& =\frac{a \mu_{i} \mu_{s}}{2\left(\mu_{i}+\mu_{s}\right)}\left[1-\exp \left(-b\left(\frac{1}{\mu_{i}}+\frac{1}{\mu_{s}}\right)\right)\right] . \quad(3.19)
\end{aligned}
$$

Similarly, for the bistatic coefficient for the diffuse reflection $\gamma_{\mathrm{L}}\left(\mu_{i}, \mu_{s}\right)$, they obtain

$$
\gamma_{\mathrm{L}}\left(\mu_{i}, \mu_{s}\right)=\frac{1}{8 \pi A \mu_{i}} \int_{\text {slab }} \mathrm{d} \boldsymbol{r}_{1} \mathrm{~d} \boldsymbol{r}_{3} F\left(\boldsymbol{r}_{1}, \boldsymbol{r}_{3}\right)
$$

$$
\times \exp \left(-\frac{\tau_{1}}{\mu_{s}}-\frac{\tau_{3}}{\mu_{i}}\right)
$$

and for cyclical diagrams

$$
\begin{aligned}
& \gamma_{\mathrm{C}}\left(\mu_{i}, \mu_{s}\right)=\frac{1}{8 \pi A_{i}} \int_{\text {slab }} \mathrm{d} \boldsymbol{r}_{1} \mathrm{~d} \boldsymbol{r}_{3} F\left(\boldsymbol{r}_{1}, \boldsymbol{r}_{3}\right) \\
& \times \exp \left(-\left(\frac{1}{2 \mu_{s}}+\frac{1}{2 \mu_{i}}\right)\left(\tau_{1}+\tau_{3}\right)\right) \\
& \times \exp \left(\mathrm{i}\left(\boldsymbol{k}_{s}+\boldsymbol{k}_{i}\right) \cdot\left(\boldsymbol{r}_{1}-\boldsymbol{r}_{3}\right)\right) .
\end{aligned}
$$

The function $F\left(\boldsymbol{r}_{1}, \boldsymbol{r}_{3}\right)$ should be solved (numerically) from Eq. (3.13). Analytical expressions for Eqs. (3.21) and (3.22) can be obtained by considering the diffusion approximation for $F\left(\boldsymbol{r}_{1}, \boldsymbol{r}_{3}\right)$. The function $F\left(\boldsymbol{r}_{1}, \boldsymbol{r}_{3}\right)$ is approximated by the diffusion propagator as given by Eq. (3.13).

The reflected intensity for a semi-infinite medium with non-absorbent particles $(a=1)$ is found to be

$$
\gamma_{\mathrm{L}}\left(\mu_{i}, \mu_{s}\right)=\frac{3 \mu_{i} \mu_{s}}{2}\left(\tau_{0}-\frac{\mu_{i} \mu_{s}}{\mu_{i}+\mu_{s}}\right)
$$

and

$$
\gamma_{\mathrm{C}}\left(\mu_{i}, \mu_{s}\right)=\frac{3}{4} \frac{1}{(\alpha+\nu)^{2}+u^{2}}\left[\frac{1}{\nu}+\frac{1-\exp \left(-2 \alpha \tau_{0}\right)}{\alpha}\right]
$$

with

$$
\begin{aligned}
& \alpha=k_{0} l_{\mathrm{sc}} \sqrt{\sin ^{2} \theta_{s}+\sin ^{2} \theta_{i}}, \quad \nu \equiv \frac{1}{2 \mu_{i}}+\frac{1}{2 \mu_{s}}, \\
& u \equiv k_{0} l_{\mathrm{sc}}\left(\mu_{i}-\mu_{s}\right), \quad l_{\mathrm{sc}} \tau_{0}=z_{0} .
\end{aligned}
$$

The scattering mean-free path is given by $l_{\mathrm{sc}}=$ $4 \pi / n|t|^{2}$ and equal to the extinction mean-free path for non-absorbent particles.

In Fig. 4, a plot of the diffusion reflected intensity as a function of the backscatter angle, for normally incident light is depicted. An increased intensity in the backscatter direction that is entirely due to the cyclical diagrams is exhibited. It is an interference contribution of counter propagating or time reversed amplitudes. The angular width of the interference contribution is inversely proportional to $k_{0} l_{\mathrm{sc}}$.

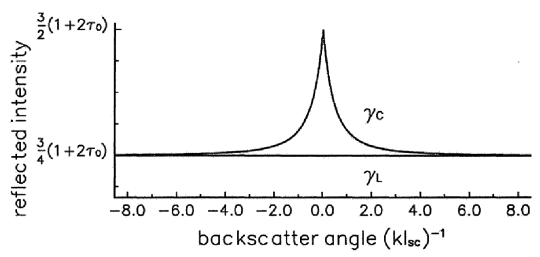

Fig. 4. Bistatic reflected intensity of a random medium.

In the exact backscatter direction $(\theta=0)$, the intensity is twice that of the diffuse background

$$
\gamma_{\mathrm{L}}(1,1)=\gamma_{\mathrm{C}}(1,1)=\frac{3}{4}\left(1+2 \tau_{0}\right) .
$$


This is an exact relation when recurrent scattering events are neglected. This can be seen as follows: if $A_{i}\left(\boldsymbol{k}_{i}, \boldsymbol{k}_{\mathrm{f}}\right)$ denotes the amplitude of a reflected wave with initial direction $\boldsymbol{k}_{i}$, first scattered at $\boldsymbol{r}_{i}$ and last scattered at $\boldsymbol{r}_{\mathrm{f}}$ in the final direction $\boldsymbol{k}_{S}$, we can write down the reflected intensity as a coherent sum over amplitudes of all possible reflection paths

$$
\begin{aligned}
& I\left(\boldsymbol{k}_{i}, \boldsymbol{k}_{S}\right)=\left|\sum_{i} A_{i}\right|^{2}=\sum_{i, j} A_{i} A_{j}^{*}=\sum_{i=j} A_{i} A_{j}^{*} \\
& +\sum_{i=-j} A_{i} A_{j}^{*}+\sum_{i=j, i \neq-j} A_{i} A_{j}^{*}
\end{aligned}
$$

The summation indices $i, j$ run over all possible paths (we have omitted the wave vector dependence of each amplitude in Eq. (3.24)). The first term on the right hand side of Eq. (3.23) is the diffuse scattered intensity: amplitude and complex conjugate travel side by side through the sample (ladder diagrams). The second term is the interference contribution where the complex conjugate follows the same path as the amplitude but in reversed order (cyclical diagrams). This is denoted by $i=-j$ in the summation. The third term yields the speckle pattern that is a wildly fluctuating (in space or direction) random intensity pattern.

If we average over the configuration of the scatterers, the speckle term drops out and we retain

$$
\langle I(\theta)\rangle=\left\langle\sum_{i}\left|A_{i}\right|^{2}\right\rangle+\left\langle\sum_{i}\left|A_{i}\right|^{2} \exp \left(\mathrm{i} \Delta \phi_{i}\right)\right\rangle
$$

where $\Delta \phi_{i}$, given by

$$
\Delta \phi_{i}\left(\boldsymbol{k}_{i}, \boldsymbol{k}_{\mathrm{f}}\right)=\left(\boldsymbol{k}_{i}+\boldsymbol{k}_{S}\right) \cdot\left(\boldsymbol{r}_{i}-\boldsymbol{r}_{\mathrm{f}}\right)
$$

is the phase difference between the direct wave $i$ and its counter-propagating partner. In the exact backscatter direction $\left(\boldsymbol{k}_{i}=-\boldsymbol{k}_{S}\right)$ the phase difference $\Delta \phi$ is zero for all paths, hence

$$
\langle I(0)\rangle=2\left\langle\sum_{i}\left|A_{i}\right|^{2}\right\rangle .
$$

For large backscatter angles, the second term of Eq. (3.11) depends on the position of the scatterers and thus averages out to zero. It follows that

$$
\langle I(\theta \gg 0)\rangle=\left\langle\sum_{i}\left|A_{i}\right|^{2}\right\rangle .
$$

Therefore, the enhancement factor, $\langle I(0)\rangle /\langle I(\theta \gg 0)\rangle$, of coherently backscattered light is exactly 2 . Absorption of the EM wave inside the disordered system does not affect the enhancement factor, because the intensity of the diffuse background and the coherently backscattered light are reduced equally. The shape of the backscatter cone will change for absorbent disordered media, the triangular shape at $\theta=0$ will be rounded off [7].

\section{On the Mishchenko decomposition}

To illustrate main points of the polarimetric decomposition for irregular media, as given in [9], a notion of target vector $\boldsymbol{k}$ is helpful,

$$
\boldsymbol{k}=V([S])=\frac{1}{2} \operatorname{Tr}([S] \Psi)=\left(k_{0}, k_{1}, k_{2}, k_{3}\right)^{\mathrm{T}},
$$

where $[S]$ is the Sinclair 2D scattering matrix defined by

$$
[S]=\left[\begin{array}{ll}
S_{11} & S_{12} \\
S_{21} & S_{22}
\end{array}\right]
$$

with radar cross-section of a given target as follows:

$$
\sigma_{q p}=4 \pi\left|S_{q p}\right|^{2}
$$

with $\boldsymbol{E}_{\mathrm{sc}}=\frac{\exp (-\mathrm{i} k r)}{r} \boldsymbol{S} \boldsymbol{E}_{\text {inc }}$. The target vector in the $\mathrm{SU}(2)$ base has the well known explicit form

$$
\begin{aligned}
& \boldsymbol{k}=\frac{1}{\sqrt{2}} \\
& \times\left[\begin{array}{llll}
S_{11}+S_{22} & S_{11}-S_{22} & S_{12}+S_{21} & \mathrm{i}\left(S_{12}-S_{21}\right)
\end{array}\right]^{\mathrm{T}} .
\end{aligned}
$$

Such a vectorization is used to generate a coherence matrix from the outer product of a vector $k$ with its conjugate transpose (or adjoint vector). In the above mentioned representation one can simply define

$$
[T]=\boldsymbol{k} \cdot \boldsymbol{k}^{* \mathrm{~T}}=T^{\dagger} .
$$
that

One considers fluctuations in the elements of (4.4) such

$$
\boldsymbol{k}=\boldsymbol{k}+\Delta \boldsymbol{k} .
$$

The coherence matrix of such a vector can be obtained from (4.5) as

$$
\begin{aligned}
T & =\boldsymbol{k}^{\dagger} \boldsymbol{k}=(\boldsymbol{k}+\Delta \boldsymbol{k}) \cdot\left(\boldsymbol{k}^{\dagger}+\Delta \boldsymbol{k}^{\dagger}\right) \\
& =\left[T_{m}\right]+\boldsymbol{k} \Delta \boldsymbol{k}^{\dagger}+\Delta \boldsymbol{k}^{\dagger} \boldsymbol{k}+\Delta \boldsymbol{k} \Delta \boldsymbol{k}^{\dagger} .
\end{aligned}
$$

We then obtain the averaged matrix $\langle T\rangle$ as

$$
\langle T\rangle=\left[T_{m}\right]+\left\langle\Delta \boldsymbol{k} \Delta \boldsymbol{k}^{\dagger}\right\rangle \text {. }
$$

From this one can define a (polarimetric) coherence matrix of fluctuations as

$$
\left\langle\Delta \boldsymbol{k} \Delta \boldsymbol{k}^{\dagger}\right\rangle=\left\langle\left[T_{\mathrm{f}}\right]\right\rangle .
$$

The starting point of the Mishchenko decomposition in [10] of that problem of multiple scattering coherence matrix par random medium are three components discussed of polarimetric EM scattering in random media. In [3] they are given by single scattering ladder and cyclical diagrams. The first is single scattering from a random distribution of particles. The second is multiple incoherent scattering due to the ladder terms in an expansion of the multiple scattering integral equations. This yields a diffuse or noncoherent background. This ladder component can be calculated using classical vector radiative transfer theory.

The third component arises from coherence (in the exact backscatter direction) between a path and its time reversed contribution. In multiple scattering terms, this contribution arises from the sum of the foregoing cyclical components in the integral equation. Mishchenko has shown that for backscatter, there is a simple but impor- 
tant relationship between the coherent and noncoherent multiple scattering contributions.

In general terms, one can expand the coherence matrix for this system as

$$
\langle T\rangle=\left\langle\left[T_{s}\right]\right\rangle+\left\langle\left[T_{\mathrm{L}}\right]\right\rangle+\left\langle\left[T_{\mathrm{C}}\right]\right\rangle=\left\langle\left[T_{s}\right]\right\rangle+\left\langle\left[T_{\mathrm{M}}\right]\right\rangle,
$$

where the subscripts $s$ stands for single scattering, L for the ladder terms, and $\mathrm{C}$ for cyclical terms. This constitutes a class of decomposition theorem based on independent scattering processes. Mishchenko obtains his important relationship between $\left\langle\left[T_{\mathrm{L}}\right]\right\rangle$ and $\left\langle\left[T_{\mathrm{C}}\right]\right\rangle$ by using the vector reciprocity theorem.

In terms of target vectors one can write the contribution to the backscattered field in the form

$$
\begin{aligned}
& \left\langle\left[T_{\mathrm{M}}\right]\right\rangle=\left\langle\left[\boldsymbol{k}_{(1, n)}+\boldsymbol{k}_{(n, 1)}\right]\left[\boldsymbol{k}_{(1, n)}^{* \mathrm{~T}}+\boldsymbol{k}_{(n, 1)}^{* \mathrm{~T}}\right]\right\rangle \\
& =\left\langle\left[\boldsymbol{k}_{(1, n)} \boldsymbol{k}_{(1, n)}^{* \mathrm{~T}}+\boldsymbol{k}_{(n, 1)} \boldsymbol{k}_{(n, 1)}^{* \mathrm{~T}}\right]\right\rangle \\
& \quad+\left\langle\left[\boldsymbol{k}_{(1, n)} \boldsymbol{k}_{(n, 1)}^{* \mathrm{~T}}+\boldsymbol{k}_{(n, 1)} \boldsymbol{k}_{(1, n)}^{* \mathrm{~T}}\right]\right\rangle \\
& =\left\langle\left[T_{\mathrm{L}}\right]\right\rangle+\left\langle\left[T_{\mathrm{C}}\right]\right\rangle,
\end{aligned}
$$

where we see that the cyclical terms arise from the cross coupling between system vectors for the two paths (these cross terms are by definition zero for the ladder contributions).

The main result follows from the observation that the two system vectors $\boldsymbol{k}_{(1, n)}$ and $\boldsymbol{k}_{(n, 1)}$ are of the form

$$
\begin{aligned}
& \boldsymbol{k}_{(1, n)}=\left(\begin{array}{llll}
k_{1} & k_{2} & k_{3} & k_{4}
\end{array}\right)^{\mathrm{T}} \Rightarrow \\
& \boldsymbol{k}_{(n, 1)}=\left(\begin{array}{llll}
k_{1} & k_{2} & -k_{3} & k_{4}
\end{array}\right)^{\mathrm{T}},
\end{aligned}
$$

where the minus sign arises since the scattering matrices for paths $(1, n)$ and $(n, 1)$ are related by the vector reciprocity theorem as

$$
\left[S_{(1, n)}\right]=\left[\begin{array}{cc}
1 & 0 \\
0 & -1
\end{array}\right]\left[S_{(n, 1)}\right]^{\mathrm{T}}\left[\begin{array}{cc}
1 & 0 \\
0 & -1
\end{array}\right]
$$

where the coherent matrix $\left[S_{(n, 1)}\right]$ is formed as a product of $n$ particle scattering matrices for the multiple scattering path (and is not necessarily symmetric).

Let us note that (4.12) simply arises from the constraint that for backscatter, the overall scattering matrix must be antisymmetric. In terms of coherence matrices it then easily follows from (4.11) and (4.12) that

$$
\begin{aligned}
& \left\langle\left[T_{\mathrm{L}}\right]\right\rangle=\left[\begin{array}{cccc}
t_{11} & t_{12} & 0 & t_{14} \\
t_{21} & t_{22} & 0 & t_{24} \\
0 & 0 & t_{33} & 0 \\
t_{41} & t_{42} & 0 & t_{44}
\end{array}\right] \Rightarrow \\
& \left\langle\left[T_{\mathrm{C}}\right]\right\rangle=\left[\begin{array}{cccc}
t_{11} & t_{12} & 0 & t_{14} \\
t_{21} & t_{22} & 0 & t_{24} \\
0 & 0 & -t_{33} & 0 \\
t_{41} & t_{42} & 0 & t_{44}
\end{array}\right] .
\end{aligned}
$$

This result ensures that the observed coherence matrix is $3 \times 3$, as expected for backscatter problems. However, from (4.14) it follows that if we can calculate the ladder terms for backscatter, then we can immediately obtain the contribution of the cyclic terms (simply by changing the sign of the $(3,3)$ element in $\langle T\rangle$. As noted earlier, the ladder terms can be calculated using conventional vector radiative transfer theory and so this result means that we can use the same theory to calculate the cyclical terms in the backscatter direction. Some implications of this result for remote sensing applications have recently been explored [10].

\section{Concluding remarks}

A concise analysis of early works on the multiple light scattering, particularly deeply penetrating the subject, was given by Kravtsov, as early as in 1993 [11]. The similar topic for random dielectrics was developed also by Orłowski and Rusek [12], which treated the problem in terms of an original, self-consistent coupled dipole model.

Up to a decade ago the multiple scattering phenomena in atmospheric researches was only associated with the two- or three body scattering features (e.g. hail flares and mirror images involving highly reflective surfaces). Recent atmospheric investigating as reported in [13] is aimed at better understanding of the water cycle and the role played by clouds and precipitation in effecting the Earth climate. Examples reported are TRRM $13.5 \mathrm{GHz}$, the CloudSat $94 \mathrm{GHz}$, the Earth CARE $94 \mathrm{GHz},(0.6 \mathrm{~mm})$, and the GPM dual 13-35 GHz $(1.6 \mathrm{~mm})$ radars. The shift towards higher radar frequencies increases the sensitivity to hydrometeors and improves the spatial resolution with reducing the size and weight of the radar system.

At $\mathrm{mm}$ wavelength, hydrometeors diffuse radiation rather isotropically compared to the visible or near-infrared region where scattering is predominantly in the forward direction. A complete understanding of radiation transport modeling and data analysis method under wide-angle multiple scattering conditions is mandatory for a correct interpretation of echoes observed by millimeters radar. The paper [13] reviews the status of research in this field. It shows the evidence of multiple scattering effects from airborne and from CloudSat observations, indicating that unique signatures observed cannot be explained by simple scattering theory. Future military application obey countermeasures systems such as decoy decors, threat assessment, reconnaissance and signal distribution.

A particular kind of enhanced backscattering for polarized EM radiation, so-called giant enhanced backscattering, has been investigated by a group of researchers [14].

It was observed that for some weakly dielectric rough surface, a significantly enhanced backscattering peak was obtained in the retroreflection direction with a ratio of peak to background as large as 10 . This is much larger than the factor of 2 predicted with cyclical diagrams contribution from time reversed partner in beam coherent backscattering. The author suggests [14] that such an 
effect may come from two contributions. One is due to the ring which always make one lay on the specular and retroreflection directions, being a result of the interference of two reflected paths which are only diffusively scattered once. It exists even in moderately rough surface and is supported only by large thickness at the film. The contrast of the ring to its background can be very large depending on the reflectivity of the rough surface.

A candidate to the other contribution to the peak is the double passage configuration, consisting in the coherent interference of a given radiation path which interacts with the rough surface at two different points with its time reversed partner.

The enhanced backscattering from very smooth conducting surfaces was also examined.

The mechanism of such phenomenon has been attributed to multiple scattering by a microstructure of the crystalline grain on the surfaces. An analogous explanation has been given for the similar behaviors of the scattered wave observed at both dielectric rough and grain-periodic surface [15-21].

Recent interesting results illustrating the role of coherent backscattering in polarimetric radar can be found in series of papers presented below, see references items from [22] to [36].

\section{Acknowledgments}

This paper is financed from sources for science in the years 2007-2010 in the frame of ordered scientific project PBZ-MNiSW-DBO-04/1/2007.

\section{References}

[1] K. Watson, J. Math. Phys. 10, 688 (1969).

[2] D. de Wolf, IEEE Trans. Antennas Propagat. AP-19, 254 (1971).

[3] L. Tsang, A. Ishimaru, J. Opt. Soc. Am. A 1, 836 (1984).

[4] N.C. Bruce, J.C. Dainty, J. Mod. Opt. 38, 579 (1990).

[5] A. Ishimaru, Proc. IEEE 79, 1359 (1991).

[6] J.B. Keler, Proc. Symp. Appl. Math. 16, 145 (1964).

[7] L. Tsang, J.A. Kong, Scattering of Electromagnetic Waves: Advanced Topics, Wiley, USA 2001.

[8] M.B. van der Mark, M.P. van Albada, A. Lagendijk, Phys. Rev. B 37, 1245 (1988).

[9] S.R. Cloude, E. Pottier, IEEE Trans. Geosci. Remote Sensing 34, 498 (1996).

[10] M.I. Mishchenko, J. Opt. Soc. Am. A 9, 978 (1992).
[11] Y. Kravtsov, Appl. Opt. 32, 268 (1993).

[12] A. Orłowski, M. Rusek, Tr. J. Phys. 23, 887 (1999).

[13] A. Baltaglia, J. Quantum Spectrosc. Radiat. Transfer 111, 917 (2010).

[14] Zu-Han Gu, Giant Enhanced Backscattering from Rough Surfaces and Related Phenomena, SOC-R783-005-1096 Final Report, 1996.

[15] H. Ogura, Z.L. Wang, Phys. Rev. B 53, 2453 (1996).

[16] M.I. Mishchenko, J.W. Hovenier, Opt. Lett. 20, 1356 (1995).

[17] M.F. Chen, A.K. Fung, Radio Sci. 23, 163 (1988).

[18] P.N. den Outer, Ph.D. Thesis, Optima Druk, Amsterdam 1995.

[19] A. DeSanto, G.S. Brown, in: Prog. in Optics XXIII, Ed. E. Wolf, Elsevier, New York 1986.

[20] H.A. Yueh, R.T. Shin, J.A. Kong, in: Prog. in Electromagnetics Research, Ed. J.A. Kong, Elsevier, New York 1989.

[21] P. Phu, A. Ishimaru, Y. Kuga, Radio Sci. 28, 533 (1993).

[22] C.C. Borel, R.E. McIntosh, IEEE Trans. Antennas Propagat. 38, 273 (1990).

[23] A. Ishimaru, J.S. Chen, P. Phu, K. Yashitomi, Waves in Random and Complex Media, 1, 591 (1991).

[24] A. Ishimaru, Y. Kuga, in: Proc. of ISAP'g2, Sapporo (Japan), Sapporo 1992, p. 563.

[25] K. Tragl, IEEE Trans. Geosci. Remote Sensing 8, 493 (1990).

[26] E. Jakeman, The effect of enhanced backscattering on target detections, NASA Grant NNX09AB3995.

[27] E.L. Gusakov, A.V. Suokov, Plasma Phys. Rep. (Russia) 29, 49 (2003).

[28] R.J. Papa, M.B. Woodworth, Backscatter Enhancement in Scattering from Rough Surface, Rept. for Jul 88-Jan 89 ADA223110, 1989.

[29] L. Ye, Chin. Phys. 15, 679 (2006).

[30] L.H. Bauner, E.H. Fooks, Proc. R. Soc. Lond. A 399, 93 (1985).

[31] K. Bouzhouane, Appl. Phys. 80, 379 (2002).

[32] C. Gruhier, Hydrol. Earth Syst. Sci. Discuss 14, 141 (2010).

[33] S. Kobayashi, Microwave Rem. Sens at the Atmuas and Environ. 5654, 106 (2004).

[34] A. Ishimaru, J. Quantum Spectrosc. Radiat. Transfer 48, 227 (1992).

[35] A. Dukata, J. Kapelewski, Acta Phys. Pol. A 114, A-67 (2008).

[36] J. Kapelewski, A. Dukata, Acta Phys. Pol. A 116, 331 (2009). 